\title{
Tratamiento Quirúrgico de los Cánceres Ováricos Derivados del Epitelio Superficial
}

\author{
Doctor Elmer Pinilla Galvis. Profesor Asociado III
}

\section{A. Conceptos generales}

Debido a que el $85 \%$ de los tumores malignos del ovario se origina en el epitelio superficial (celómico, mesotelial o germinal) que recubre el órgano, hay mayor experiencia en el manejo de ellos. Las neoplasias malignas originadas en las otras estructuras ováricas (estroma de los cordones sexuales, células germinales, tejidos inespecíficos) son bastante raras. Debido a ello, los estudios hechos por distintos autores para establecer la mejor pauta de tratamiento tropiezan con dificultades para investigaciones aleatorias $y$, como resultado, no se tiene un manejo consensual.

El tratamiento básico inicial de los tumores ováricos malignos derivados del epitelio celómico es quirúrgico, y éste depende del estado cl ínico, del tipo histológico, del grado de anaplasia y de la edad de la paciente. Dadas las dificultades para hacer el diagnostico antes de la cirugía, la mayor parte de los casos se descubre en el momento de la laparatomía con la toma de biopsias para estudio por congelación. Importa recalcar aquí que los informes de tales biopsias no solamente deben enunciar si hay o no malignidad, sino que deben incluir el tipo histológico del tumor, la presencia o no de otros tipos celulares y el grado de diferenciación.

\footnotetext{
* Facultad de Medicina, Universidad de Antio. quia Medellín.
}

El ginecólogo debe tener un conocimiento profundo tanto de la clasificación clínica de las etapas de progresión del cáncer ovárico, como del comportamiento de los distintos tipos de ellos, de sus índices de bilateralidad, de sus grados de anaplasia y de su sensibilidad a la radio y a la quimioterapia, porque, especialmente en mujeres jóvenes, que desean conservar su capacidad reproductora, tales conocimientos son los que van a decidir el tratamiento adecuado.

No es necesario recalcar la importancia de una historia clínica completa. Además, de los exámenes de laboratorio rutinarios (hematimetría, radiografía de tórax, placa simple de abdómen, etc.), muchos autores recomiendan tener un estudio histológico del endometrio para descartar una neoplasia concomitante, primaria o metastásica. Los estudios radiológicos de vías digestivas y el examen cuidadoso de las mamas pueden descubrir un cáncer insospechado que de metástasis al ovario. La urografía excretora informa del estado de las vías urinarias.

El método quirúrgico de base para los tumores derivados del epitelio celómico consiste en ovariectom ía bilateral, histerectom ía, salpingectom ía bilateral, omentectomía y resección tumoral lo más completamente posible. Hay autores que recomiendan la apendicectomía.

Deben extirparse ambos ovarios por la alta frecuencia de bilateralidad. No solamerite el cáncer de un ovario se propaga 
al otro por vía linfática sino por medio del líquido peritoneal. En los últimos años se ha venido hablando de la "carcinogénesis de campo", teoría ésta que explica la presencia de un segundo primario en la gonada opuesta diciendo que los ovarios son un sólo órgano, que ambos están sometidos a unas mismas influencias $y$ a unos mismos estímulos de un mismo medio ambiente y que, lo que origina la formación de una neoplasia en uno de ellos, origina la misma en el otro.

La histerectomía está indicada por la alta frecuencia de compromiso tubario. Además, las relaciones del útero con el fondo de saco de Douglas, sitio al cual descaman las células malignas desprendidas del ovario que circulan por el líquido peritoneal, hacen del órgano un sitio de metástasis frecuentes. Por otra parte, el endometrio también frecuentemente muestra compromisos tumorales.

La omentectomía está indicada porque el epiplón es uno de los primeros lugares de propagación temprana. En los estados iniciales, a pesar de que macroscópicamente puede no verse invasión tumoral, el porcentaje de siembras microscópicas es alto y su descubrimiento es importante para la correcta clasificación clínica y para considerar el manejo ulterior de las zonas de riesgo metastásico. Sin embargo, no está exenta de problemas ya que puede provocar adherencias intestinales a la pared anterior del abdómen, que dificultan las exploraciones posteriores; aumenta las probabilidades de daño intestinal por la radioterapia posterior. La omentectomía es indispensable cuando se planea la instilación peritoneal de coloides radiactivos pues el epiplón impide la distribución uniforme de esas sustancias y provoca su acumulación en los lugares de declive como los fondos de saco. En general, basta con la omentectom ía subtotal a partir del colon transverso.
La resección tumoral debe ser lo más completamente posible pero no se deben hacer resecciones indiscriminadas que rebasen los límites del sentido común. Las resecciones irracionales comprometen el bienestar de las pacientes, demoran su recuperación y retardan la terapia adyuvante ulterior. Las resecciones y reanastomosis intestinales se justifican solamente cuando, al extirpar el sitio metastásico, se elimina todo el tumor macroscópico. Muchas veces las siembras metastásicas son por "implantes" y no por penetración de las paredes intestinales $y$, entonces, es posible desprenderlas sin causar mayores daños a las paredes de la víscera.

Quienes recomiendan la apendicectomía rutinaria sostienen que el apéndice es un sitio de frecuentes metástasis precoces.

\section{B. Tipo de Incisión}

De capital importancia es practicar una exploración completa de la cavidad abdominal porque de ella depende la correcta formulación de la etapa cl ínica, que, a su vez, es el factor que mayormente interviene en la decisión del tratamiento que se debe seguir. Para lograr esto, es indispensable una incisión longitudinal amplia, susceptible de prolongar, que permita reconocer por palpación $y$ por visualización todos los órganos $y$ regiones abdominales $\mathrm{y}$ tomar biopsias de los sitios sospechosos: fondo de saco de Douglas, vejiga, genitales internos, epiplón, apéndice, intestinos, mesenterio, hígado, estómago,.superficie inferior del diafragma, cadenas ganglionares retroperitoneales y goteras perietocólicas. $\mathrm{Si}$ esta exploración no es minuciosa, es probable la subclasificación de la etapa clínica y los tratamientos inadecuados que llevan al fracaso terapéutico. Todas esas maniobras no son posibles cuando la apertura del abdómen se hace mediante 
incisiones transversales, adecuadas para otros tipos de patología pélvica, pero no para patología tumoral ovárica, pues en este último caso se sacrifica la correcta exploración abdominal en pro de posibles menores riesgos de eventraciones $y$ del aspecto estético. Day y Smith indican la toma rutinaria de biopsias múltiples de toda la superficie peritoneal, de las goteras perietocólicas, de la cara infe rior del diafragma, del epiplón, de los ganglios retroperitoneales y de las superficies de la pelvis. McGowan recomienda, antes de la apertura total del peritoneo, abrir un pequeño ojal en éste $y$ tomar por aspiración una muestra de líquido peritoneal o de ascitis para estudio citológico. Con esa maniobra se evita la contaminación hemática de la muestra. Este autor no recomienda el "lavado" ya que la población celular queda notablemente disminuida por unidad de volúmen, y, además, siempre es posible hallar aunque sea una muestra en los sitios de declive en los que se acumula el líquido peritoneal como el Douglas y las goteras perietocólicas.

\section{Tratamiento conservador}

Más o menos el $7 \%$ de los casos de tumores malignos del ovario ocurren en mujeres menores de 35 años. Cuando la enfermedad se halla localizada en un sólo ovario, la conducta que se debe seguir tiene que ajustarse no solamente a ese hecho, sino al tipo del tumor y a su grado de diferenciación, y a los deseos y criterios de la paciente. Tanto ella como el ginecólogo deben comparar los beneficios de la maternidad contra los riesgos de un posible tratamiento insuficiente. Irónicamente, W.G. Smith considera que la conducta de preservar el ovario contralateral y el útero es, a la inversa, otra forma "radical" de tratamiento. Es posible que los términos "radical" $y$ "conservador" en la cirugía de los tumores malignos del ovario sean, en el mejor de los casos, términos equívocos. Debido a que muchas veces se debe y se tiene que dejar gran parte del tumor y las cadenas ganglionares de drenaje, no cabe hablar de cirugía "radical" así se hayan extirpado el útero, los ovarios, el epiplón y gran parte del tumor. En otras ocasiones sólo es posible tomar una simple biopsia, en cuyo caso tampoco es lógico hablar de cirugía "conservadora".

En un estudio de 190 casos de cáncer ovárico limitado a un sólo ovario, Munnell no pudo comprobar diferencias estadísticas de mortalidad entre los casos tratados con ovariectomía ipsolateral $y$ los tratados con ovariectomía bilateral. Sin embargo, la supervivencia a 5 años fue de 74 y $79 \%$ respectivamente.

Se tiene ya el suficiente conocimiento de que la supervivencia aumenta considerablemente en casos de tumores de grado bajo de anaplasia y en lo "lim ítrofes", y que, en cambio, ella disminuye verticalmente en los anaplásicos. En vista de esto, Smith opina que con estos últimos tumores no caben consideraciones de edad ni de deseos obstétricos y que se debe, extirpar los genitales internos.

Los tumores malignos epiteliales se presentan bilateralmente en $30 \%$ de los casos, pero algunos tipos celulares y grados pueden hacerlo hasta en $50 \%$. Por ello, muchos autores consideran que conservar un ovario potencialmente maligno es un riesgo absolutamente inaceptable.

La inspección macroscópica y la palpación son métodos a todas luces insuficientes para descartar compromiso $\mathrm{mi}$ croscópico. Se sabe que entre 12 y $18 \%$ de los ovarios contralaterales de aspecto "sano" tienen focos microscópicos de células malignas. Si la gonada contralateral no muestra enfermedad microscópica, de todas maneras al dejarla "in situ" irá a estar sometida a los mismos factores que provocaron la aparición del tumor 
maligno en la otra, lo cual aumenta el riesgo de desarrollo ulterior de cáncer en ella.

En ocasiones hay dudas en los informes de las biopsias por congelación tanto del ovario enfermo como del contralateral. En cualquiera de los dos casos, se debe cerrar el abdómen sin extirpar el útero ni el ovario contralateral, esperar el informe definitivo y proceder según él.

En las pacieıtes añosas resulta inconsecuente hacer consideraciones de estado precoz, de tipo de tumor y de grado de anaplasia. La indicación en ellas es la cirugía completa.

\section{Clasificación clínica}

I (Ovarios)

II (Pelvis)

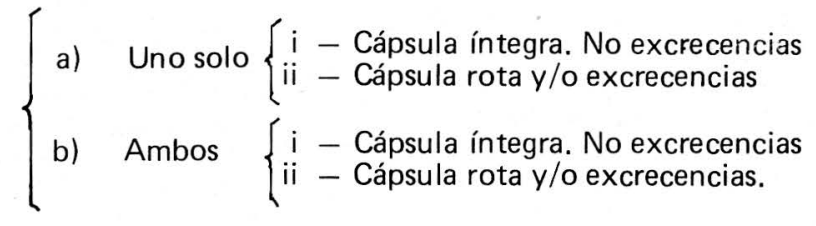

c) a o $\underline{b}$ con ascitis

a) Utero y/o trompas

b) Otros órganos pélvicos

c) a_o b con ascitis

III (Abdómen) Ganglios retroperitoneales, intestinoepiplón. Excepto hígado.

IV Metástasis a distancia. Incluso hígado.

\section{E. Cirugía según los Estados Clínicos}

\section{Estado lai}

Presentamos a continuación el criterio de Grenwald sobre qué se debe extirpar

1. Tumor diferenciado o "lim ítrofe" en este estado clínico y luego mostraremos las divergencias de otros autores.

\section{Jóvenes}

Citología del líquido peritoneal

Exploración abdominal completa

Coforectomía ipsolateral

Biopsia del ovario contralateral

\section{Añosas}

Idem

Idem

Ooforectomía bilateral

Histerectomía

Omentectom ía

Apendicectomía 
No se ha establecido qué terapia posterior se pueda seguir después de este tratamiento quirúrgico.

\section{Tumor anaplásico:}

\author{
Jóvenes \\ Con interés obstétrico \\ Citología del l íquido peritoneal \\ Exploración abdominal completa \\ Ooforectomía ipsolateral \\ Biopsia de ovario contralateral \\ Embarazo \\ ¿Completar cirugía? \\ ¿Radioterapia?
}

Malkasian, en la Clínica Mayo, no parece tomar en cuenta el grado de anaplasia: en pacientes jóvenes que desean procrear, extirpa el ovario enfermo; y en las pacientes que no lo desean o son añosas, hace la cirugía "completa" y no indica ningún tipo de terapia adicional.

McGowan aconseja linfadenectomía aórtica en casos de enfermedad inicial.

Dado que el carcinoma endometrioide, el carcinoma de células claras y el cistadenocarcinoma mucinoso tienen escaso potencial maligno, Kottmeier, citado por Parsons-Sommers, aconseja ovariectomía ipsolateral y biopsia del ovario contralateral, conducta con la que no concuerdan los tratadistas mencionados quienes dejan el ovario restante y el útero sólo en casos de que la biopsia del ovario "sano" sea negativa o dudosa, en cuyo caso cierran el abdómen esperan el resultado definitivo y completan la cirugía si el informe es desfavorable. Ellos tampoco parecen tener en cuenta el grado del tumor; pero, en pacientes jóvenes que desean procrear, indican que esos tres tumores constituyen "la única indicación de cirugía conservadora".

\author{
Añosas o \\ Jóvenes sin interés \\ Idem \\ Idem \\ Ooforectomía bilateral \\ Histerectom ía \\ Omentectomía \\ Apendicectomía \\ ¿Radioterapia?
}

En nuestro medio (Hospital Universitario San Vicente de Paúl, Medellín) el doctor Jaime Botero indica la conservación de la gonada contralateral y el útero en casos lai de tumores de grado bajo o "en los límites" en pacientes jóvenes en general. $Y$ respecto de la terapia adyuvante ulterior, el doctor Augusto Llamas, jefe de Radioterapia de esa institución, en una conversación personal con el autor de esta revisión, se muestra partidario de radioterapia y de quimioterapia en todos los casos de lb a III. Darío Méndez, radioterapeuta del mismo centro científico y docente, piensa que tal conducta está justificada también en casos de tumores anaplásicos en estado la.

Cuando se trata de Disgerminomas, el patólogo debe, mediante cortes multiples, examinar todo el tumor para descartar la presencia de otros tipos tumorales derivados de las células germinales (coriocarcinoma, teratoma maligno, tumor del seno endodérmicol, pues estas mezclas le confieren al Disgerminoma la malignidad correspondiente al otro tumor que, como se sabe, es mucho mayor, 
y el tratamiento y el pronóstico varía notablemente.

\section{Estado laii}

Greenwald supone que ya hay diseminación de células malignas en toda la cavidad adbominal que "aumenta la posibilidad de recidivas", por lo cual aconseja radioterapia complementaria. Aquí no toma en cuenta el líquido peritoneal; tampoco hace caso de la edad de la paciente:

\section{Exploración abdominal completa \\ Ovariectomía bilateral \\ Histerectomía \\ Omentectomía \\ Apendicectomía \\ Radioterapia (Cobalto o radionúclidos)}

Greenwald es, de los autores revisados, el único que se ocupa del estado laii, a excepción de Malkasian, quien concuerda con la conducta de Greenwald pero en cuanto a la terapia adyuvante posterior a la cirugia cree que, si en lugar de la radio se escoge la quimioterapia, ella sólo se prescribirá durante 6 meses.

\section{Estados Ia anaplásicos, Ib, Ic.}

Para McGowan, la conducta que se debe tomar es:

Exploración abdominal completa

Ovariectomía bilateral

Histerectomía

Omentectomía

Apendicectomía

Quimioterapia (Melfalán)

o

Radioterapia con coloides
Malkasian, en Ib y Ic, toma igual conducta quirúrgica. En la también, sin tener en cuenta el grado de anaplasia. La quimioterapia la indica por 6 meses. Barber aconseja Radionúclidos; la quimioterapia para él es opcional (?), después de los radioisótopos.

Estados IIa, IIb, IIc

Greenwald toma la siguiente conducta a partir del estado $\mathrm{lb}$ :

Exploración abdominal completa

Ovariectomía bilateral

Histerectomía

Omentectomía

Apendicectomía

Resección de implantes tumorales (si los hay).

Radioterapia

Quimioterapia (si se sospecha tumor residual después de radioterapia).

McGowan toma igual conducta quirúrqica e indica quimioterapia sola o con radioterapia. Malkasian, después de la cirugía, adopta quimioterapia durante 12 meses al cabo de los cuales practica "la segunda mirada": si no se encuentra nada, se suspende la quimioterapia. Barber opina que los radionúclidos son "opcionales" y que debe haber quimioterapia.

\section{Estado III}

Greenwald opina que se debe resecar todo lo que racionalmente sea resecable y que, en ocasiones, sólo es posible tomar biopsias. Después de la cirugía, las pacientes pueden quedar en:

1 Malas condiciones: tratamiento paliativo.

2 Buenas condiciones: 
a) OPTIMAS (IIla): tumor residual menor de $2 \mathrm{cms}$ Radioterapia Quimioterapia (incluso sin masa palpable).

b) SUBOPTIMAS (IIlb): tumor residual mayor de $2 \mathrm{cms}$ o tumor de células germinativas (excepto disger minoma)

Quimioterapia

(se interrumpe al desaparecer tumor palpable)

Radiote rapia (excepto demás tumores de células germinativas)

Quimioterapia

McGowan concuerda con el enfrentamiento quirúrgico luego del cual prescribe quimioterapia. Este autor afirma que la radioterapia ha sido abandonada en casi todas partes en estados III y IV de tumores epiteliales y dice que actualmente se investiga con Melfalán sólo o en combinación con inmunoterápicos (BCG, células tumorales irradiadas o tratadas con neuramidaza, corinebacterium parvum, levamizole). En los casos subóptimos se investiga con hexametilenmelamina más Melfalán, y adriamicina más ciclofosfamida. Parsons-Sommers estiman conveniente hacer colostomía en caso de inminencia de obstrucción intestinal y recomiendan la radioterapia adyuvante. Barber concuerda con McGowan en cuanto al abandono de la radioterapia, pues las dosis cancericidas provocan daño hepato-renal, y aconsejan quimioterapia. Malkasian adopta quimioterapia y reexploración a los 24 meses:

Si no tumor residual, suspender

quimioterapia

Si sí tumor residual, seguir

quimioterapia
Radioterapia en casos de enfermedad localizada progresiva a pesar de quimioterapia.

\section{Estado IV}

Greenwald recomienda quimioterapia. Barber, la resección de lo racionalmente resecable, quimioterapia posterior y radionúclidos si hay nódulos residuales menores de $2 \mathrm{cms}$. Malkasian tiene igual conducta que para el estado III, así como McGowan.

\section{Radioterapia preoperatoria}

Según Parsons-Sommers, algunos autores estiman conveniente, en los estados II y III, iniciar el tratamiento con radio terapia preoperatoria para atacar las vías de diseminación y hacer más fácil los desprendimientos tumorales durante la cirugía. Kottmeier, según los tratadistas, en casos de carcinoma seroso en estado II y en mujeres jóvenes recomienda, ovariectomía ipsolateral, luego radioterapia y después completar la cirugía. Pero si el tumor es endometrioide, de células claras, o mucinoso, y la paciente desea más hijos, sólo extirpa el ovario comprometido. Los doctores Llamas y Méndez no concuerdan con la radioterapia preoperatoria.

\section{F. La segunda mirada}

Las reoperaciones fueron popularizadas por Wangestein en 1957 para pacientes de cáncer de colon y de estómago. La maniobra tiene un destacado lugar en el tratamiento del cáncer del ovario. Como lo sugiere su nombre, se practica luego de un primer intento quirúrgico seguido de radioquimioterapia, y su finalidad es hacer una "limpieza" de residuos tumorales. Además, puede decidir la suspen- 
sión o el cambio de quimioterápicos. Las indicaciones, según Greenwald, son:

1. Supervivencia de 24 meses. Si no hay restos tumorales, se suspende la quimioterapia. Si los hay, se reseca lo que racionalmente sea resecable.

2. Leuco y trombocitopenias notables antes de 24 meses sin enfermedad residual detectable. Según haya o no tejido tumoral, se procede en concordancia.

3. Cuando en la primera cirugía el tumor no fue extirpado pero se hace móvil y "libre" con la radio y la quimioterapia. Se extirpa lo racionalmente resecable y se continúa con radioterapia (si no se hizo antes) y con la quimioterapia.
En la cirugía de "segunda mirada" se debe explorar toda la cavidad abdominal y tomar biopsias de los sitios y de los ganglios sospechosos. No se consideran "de segunda mirada" las intervenciones cuyos únicos fines sean, por ejemplo, una colostomía.

\section{Resumen}

Se revisan los criterios de 15 autores sobre manejo quirúrgico del cáncer ovárico originado en el epitelo celómico entre 1975 y 1980. Se hace notar la importancia de que el ginecólogo domine el conocimiento de los estados clínicos, de los distintos tipos de tumores, de su conducta biológica, de su grado de anaplasia y de su respuesta a la terapia adyuvante especialmente en mujeres jóvenes que desean procrear.

\section{SURGICAL TREATMENT OF OVARIAN CARCINOMAS DERIVED FROM THE SUPERFICIAL EPITHELIUM}

\section{Summary}

A review is made of the criteria of 15 authors on surgical treatment of ovarian carcinoma originated in the superficial epithelium between 1975 and 1980. It is of outmost importance that the gyneco- logist know the clinical reports, the ditterent types of tumors, their biological behavior, their degree of anaplasia and their response to adyuvant therapy, specially in young women wishing to procreate.

\section{Bibliografia}

1. BARBER, H.R.K. "Radiation therapy". Manual of Gynecologic Oncology. J.B., Lippincott Company. Philadelphia-Toronto, 1980.

2. BOTERO, J. "Tumores del ovario". Ginecología-Obstetricia. Texto integrado., U. de A. Medellín. Seg. edic. 1980.

3. CLARK, D.G.C. et al. "Tratamiento del cáncer ovárico". Ginecol. Obst. Temas actuales. Interarnericana. Abril 1976.

4 GREENWALD, E.F. "Tumores del ovario". Clin. Obst. Gin. Interamericana. Dic 1975.

5. HUDSDN, C.N. "Lugar de la cirugía en el tratamiento del cáncer ovárico". Ginec. Obst. Temas actuales. Interamericana. Vol. 3, 1978.

6. JULIAN, C.G. "Neoplasia epitelial germinal del ovario". Clin. Obst. Gin. Interamericana, Marzo/74. 
7. McGOWAN, L. Gynecologic Oncology. Appleton Century Crofts New York.

8. MALKASIAN, G.D. et al. “Histología de los tumores epiteliales del ovario: utilidad clínica y significación pronóstica de la clasificación y grados histológicos". Seminarios de Oncología-De Vita. Cáncer del ovario. Edit. Médica Panamericana, 1976;

9. DAY, T.G. et al. “Diagnóstico y determinación de etapas en el carcinoma de ovario". Seminarios de Oncología-De Vita,. Edit. médica Panamericana. 1976.

10. WALLACH, R.C. et al. "Importancia de los procedimientos quirúrgicos de revisión en la determinación de etapas y tratamiento del carcinoma del ovario". Seminarios de Oncología-De Vita. Edit. Médica Panamericana, 1976.
11. RUBIN, Ph. "El problema de subestimar la etapa del cáncer de ovario". Seminarios de Oncología-De Vita. Edit. Médica Panamericana, 1976.

12. NELSON, J.H. Atlas of Radcal pelvic surgery. Appleton century crofts. Second edition 1977.

13 PARSONS-SOMMERS. Gynecology. W. Saunders company, 1978.

14. SMITH, W.G. "Tratamıento quirúrgico del carcinoma ovárico epitelial". Clin. Obst. Ginec. Interamericana, Vol. 4, 1979.

15. WATRING, W.G. et al. "Exámenes de selección y diagnóstico en cáncer de ovario". Clin Obst. Ginec. Interamericana. Vol. $4 / 79$ 